Erika Wagner (Hrsg)

\title{
Grundfragen des Erwachsenenschutzrechts
}


Erika Wagner (Hrsg)

Grundfragen des

Erwachsenenschutzrechts

2019

Handbuch 
Univ.-Prof. ${ }^{\text {in }}$ Dr. ${ }^{\text {in }}$ Erika Wagner

Institutsvorständin des Instituts für Umweltrecht, Abteilungsleiterin der Abteilung Umweltprivatrecht am Institut für Zivilrecht, JKU Linz

Das Werk ist urheberrechtlich geschützt.

Die dadurch begründeten Rechte, insbesondere die der Übersetzung, des Nachdruckes, der Entnahme von Abbildungen, der Funksendung, der Wiedergabe auf fotomechanischem oder ähnlichem Wege und der Speicherung in Datenverarbeitungsanlagen, bleiben, auch bei nur auszugsweiser Verwertung, vorbehalten. Die Wiedergabe von Gebrauchsnamen, Handelsnamen, Warenbezeichnungen usw. in diesem Buch berechtigt auch ohne besondere Kennzeichnung nicht zu der Annahme, dass solche Namen im Sinne der Warenzeichen- und Markenschutz-Gesetzgebung als frei zu betrachten wären und daher von jedermann benutzt werden dürfen.

Produkthaftung: Sämtliche Angaben in diesem Fachbuch/wissenschaftlichen Werk erfolgen trotz sorgfältiger Bearbeitung und Kontrolle ohne Gewähr. Eine Haftung der Herausgeberin, der AutorInnen oder des Verlages aus dem Inhalt dieses Werkes ist ausgeschlossen.

\section{(C) 2019 Verlag Österreich GmbH, Wien}

www.verlagoesterreich.at

Gedruckt in Deutschland

Satz: abavo GmbH, 86807 Buchloe, Deutschland

Druck: Strauss GmbH, 69509 Mörlenbach, Deutschland

Gedruckt auf säurefreiem, chlorfrei gebleichtem Papier

https://doi.org/10.33196/9783704683588

Bibliografische Information der Deutschen Nationalbibliothek

Die Deutsche Nationalbibliothek verzeichnet diese Publikation in der Deutschen Nationalbibliografie; detaillierte bibliografische Daten sind im Internet über http://dnb.d-nb.de abrufbar.

ISBN 978-3-7046-8257-4 Verlag Österreich 


\title{
Die Haftung des Erwachsenenvertreters
}

\author{
Erika Wagner/Lydia Burgstaller
}

\section{Einleitung}

Vorweg seien die wesentlichen Begriffe in Erinnerung gerufen: Schutzberechtigte Personen weisen Defizite im Rahmen der sog Geschäftsfähigkeit auf: Dies ist die Fähigkeit einer Person, durch eigenes rechtsgeschäftliches Handeln Rechte und Pflichten zu begründen. Die Geschäftsfähigkeit ist eine besondere Form der Handlungsfähigkeit, zugeschnitten auf den Bereich der Rechtsgeschäfte. Personen, die sich aus anderen Gründen als der Minderjährigkeit nicht durch eigenes Handeln berechtigen und verpflichten können, sind nicht handlungsfähig. Handlungsfähigkeit setzt wiederum Entscheidungsfähigkeit voraus ( $\mathbb{2} 24 \mathrm{Abs} 1 \mathrm{ABGB}$ ). Entscheidungsfähig ist, wer die Bedeutung und die Folgen seines Handels im jeweiligen Zusammenhang verstehen, seinen Willen danach bestimmen und sich dementsprechend verhalten kann. Um diese Defizite betroffener Personen - vor dem Hintergrund der UN-Behindertenkonvention - bestmöglich zu kompensieren, hat der Gesetzgeber das Recht der Sachwalterschaft bereits grundlegend novelliert. ${ }^{2}$ Nicht alle Fragen wurden dabei - trotz der überwiegend positiven Grundbewertung der Neuregelung - abschließend gelöst.

Schon im Rahmen der alten Rechtslage wurden Fragen der schadenersatzrechtlichen Haftung des Sachwalters bzw des Erwachsenenvertreters/Vorsorgebevollmächtigten in der Rsp ${ }^{3}$ eher vernachlässigt. Grund dafür könnte sein, dass Schäden, welche ein Sachwalter schuldhaft zugefügt hat, oftmals mangels hinreichender Kontrolle verborgen blieben oder es zu einer gütlichen Einigung kam, da (va bei Sachwaltervereinen) eine Haftpflichtversiche-

1 Übereinkommen über die Rechte von Menschen mit Behinderungen sowie das Fakultativprotokoll zum Übereinkommen über die Rechte von Menschen mit Behinderungen, BGBl III 2008/155.

2 Sachwalterrechts-Änderungsgesetz 2006, BGBl I 2005/43.

3 Mit der persönlichen Haftung des Sachwalters befasste sich der OGH inhaltlich bisher in folgenden Fällen: OGH 28.2.1989, 2 Ob 8/89; 22.10.2001, 1 Ob 197/01d, JBl 2002, $304 ; 17.3 .2004,7 \mathrm{Ob} 11 / 04 z$. 
rung vorlag. ${ }^{4}$ Dennoch ist klar - wie auch der OGH in den wenigen entschiedenen Fällen ${ }^{5}$ betont -, dass der Vertreter/die Vertreterin nach „allgemeinen schadenersatzrechtlichen Grundsätzen" zu haften hat.

Der vorliegende Beitrag nimmt die Neuerungen des 2. Erwachsenenschutz-Gesetzes ${ }^{6}$ zum Anlass, sich mit Fragen der Haftung des Erwachsenenvertreters zu beschäftigen und geht dabei der Frage nach, ob es durch die neue Rechtslage zu Änderungen in Bezug auf die Haftung des Erwachsenenvertreters bzw Vorsorgebevollmächtigten gekommen ist.

\section{A. Selbstbestimmung als Kern des Persönlichkeitsrechts}

Mit der Einführung des 2. ErwSchG soll die Selbstbestimmung von Menschen, die aufgrund einer psychischen Krankheit oder wegen einer vergleichbaren Beeinträchtigung in ihrer Entscheidungsfähigkeit eingeschränkt sind, gefördert werden. Nach der Intention des Gesetzgebers ${ }^{7}$ sollen diese - soweit möglich - selbst über ihre rechtlichen Beziehungen bestimmen können. Im Rahmen dieser Novellierung wurde das bisherige System der Sachwalterschaft erneut und grundlegend reformiert. Es wurden/neue Vertretungsmodelle und Alternativen eingeführt, die Autonomie im Rechtsverkehr und in persönlichen Angelegenheiten gestärkt und - neben weiteren Inhalten (Personensorge, Erwachsenenschutzvereine, Verfahrensrecht, Berufsrecht der Notare und Rechtsanwälte etc) - zêitgemäße terminologische Anpassungen vorgenommen. ${ }^{8}$ Das neue System der Erwachsenenvertretung fußt nun im Kern auf vier Säulen:

- der Vorsorgevollmacht,

- der gesetzlichen Erwachsenenvertretung,

- der gewählten Erwachsenvertretung und

- der gerichtlichen Erwachsenenvertretung.

Die Frage nach der Verantwortung im Falle einer Schädigung von Rechtsgütern der schutzberechtigen Person ist dabei grundsätzlich in allen Kategorien die gleiche, wobei im Detail aber die Pflichten der jeweiligen Vertreter andere sein mögen.

4 Volgger, Die Haftung des Sachwalters. Ein Überblick mit Beispielen aus der Rechtsprechung, iFamZ 2007, 74.

$5 \mathrm{Vgl} \mathrm{zB} 1 \mathrm{Ob} 197 / 01 \mathrm{~d}$.

6 BGBl I 2017/59; in der Folge: 2. ErwSchG.

7 ErläutRV 1461 BlgNR 25. GP, 1.

$8 \mathrm{Vgl}$ einen Überblick über die Neuerungen des ErwSchG bietend Kathrein in DeixlerHübner/Schauer (Hrsg), Erwachsenenschutzrecht. Beiträge zum 2. Erwachsenenschutzgesetz (2018) 1 ff; ebenso Barth (Hrsg), Das neue Erwachsenenschutzrecht (2017) $1 \mathrm{ff}$. 
Grundsatz und oberste Priorität der Neukodifikation im Rahmen des 2. ErwSchG ist - wie bereits erwähnt - die Erhaltung der Autonomie der betroffenen Personen. ${ }^{9}$ Insofern halten die Grundsatzbestimmungen der \239 ff fest, dass im rechtlichen Verkehr dafür Sorge zu tragen ist, dass volljährige Personen, die aufgrund einer psychischen Krankheit oder einer vergleichbaren Beeinträchtigung in ihrer Entscheidungsfähigkeit eingeschränkt sind, möglichst selbständig, erforderlichenfalls mit entsprechender Unterstützung, ihre Angelegenheiten selbst besorgen können. Dem Grundsatz der möglichst weitgehenden Autonomie ${ }^{10}$ folgend statuiert $\ 239$ die Subsidiarität der diversen Vertretungsarten. Keine der vier Vertretungsarten soll nach dem Konzept des 2. ErwSchG - anders als nach alter Rechtslage - automatisch zum Verlust der Handlungsfähigkeit der vertretenen Person führen. ${ }^{11}$

Diese Subsidiarität der Vertretung kann auch als die Kehrseite des Primats der Selbstbestimmung gesehen werden. ${ }^{12}$ Daraus folgt klarerweise, dass der Geschäftsverkehrs bewusst dem Risiko ausgesetzt wird, dass uU wegen fehlender Geschäftsfähigkeit des Handelnden kein Vertrag zustande kommt. Allein aus diesem Umstand lassen sich haftungsrechtliche Folgen aber weder gegen einen allenfalls bestellten Vertreter wegen Unterlassung der Vertretung und schon gar nicht gegen die schutzberechtigte Person ableiten.

Selbst wenn nämlich ein Erwachsenenvertreter bestellt sein sollte, hat dessen Pflicht, den Schutzberechtigten bei seinen Angelegenheiten zu unterstützen und ihn - gegebenenfalls zu vertreten - idR keine Außenwirkung. ${ }^{13}$ Aus dem Nichtzustandekommen eines Rechtsgeschäfts, das der Betroffene abzuschließen vermeinte (was mangels Geschäftsfähigkeit aber scheiterte), lässt sich idR keine Haftung Dritten gegenüber ableiten. Der OGH hat bereits zur Sachwalterschaft folgende Position vertreten: Die vom Sachwalter zu beachtenden Verhaltenspflichten bezwecken nur den Schutz des Betroffenen vor Rechtsnachteilen als Subjekt der Sachwalterschaft, so insb auch vor allfälligen Vermögensnachteilen. Deren Zweck ist es dagegen nicht, Dritte vor Vermögensschäden zu bewahren, die sie im direkten rechtlichen Verkehr mit dem Betroffenen aufgrund eigener Nachlässigkeit erleiden. ${ }^{14}$ Das gilt im Bereich des 2. ErwSchG umso mehr. ${ }^{15}$ Dessen gesetzliche Wertung ist es immerhin,

9 ErläutRV 1461 BlgNR 25. GP, 17.

10 Katbrein in Deixler-Hübner/Schaner, HB Erwachsenenschutzrecht (2018) Rz 1.18 ff; ebenso Kathrein in Deixler-Hübner/Schauer, Erwachsenenschutzrecht. Beiträge zum 2. Erwachsenenschutz-Gesetz $8 \mathrm{ff}$.

11 ErläutRV 1461 BlgNR 25. GP, 5 und 20, Abschaffung des konstitutiven Verlustes der Geschäftsfähigkeit einer durch Sachwalter vertretenen Person; zur Handlungsfähigkeit vgl auch Barth in Deixler-Hübner/Schauer, Erwachsenenschutzrecht. Beiträge zum 2. Erwachsenenschutz-Gesetz $20 \mathrm{f}$.

12 Schaner, Die vier Säulen des Erwachsenenschutzrechts, iFamZ 2017/148, 150.

13 Riedler, ZAK Spezial - Erwachsenenschutzgesetz (2018) 48 f; Barth in Deixler-Hübner/ Schauer, Erwachsenenschutzrecht. Beiträge zum 2. Erwachsenenschutz-Gesetz 23.

$141 \mathrm{Ob} \mathrm{197/01d.}$

15 Bollenberger in Deixler-Hübner/Schauer, HB Erwachsenenschutzrecht Rz 10.9. 
dass betroffene Personen nur dann durch einen Vertreter am Rechtsverkehr teilnehmen sollen, wenn sie dies selbst vorsehen oder eine Vertretung zur Wahrung ihrer Rechte und Interessen unvermeidlich ist.

Ein Umkehrschluss, dass sich Dritte im Falle der fehlenden Geschäftsfähigkeit des Betroffenen auf die Untätigkeit des Vertreters berufen und diesen haftbar machen könnten, folgt daraus nicht. Soweit eine volljährige Person bei Besorgung ihrer Angelegenheiten entsprechend unterstützt wird oder selbst - besonders durch eine Vorsorgevollmacht - für deren Besorgung im erforderlichen Ausmaß vorgesorgt hat, darf für sie kein Erwachsenenvertreter tätig werden. Dies spielt im Rahmen des Vorgangs zur Bestellung eines Erwachsenenvertreters eine Rolle. ${ }^{16}$ Die Bestellung eines Erwachsenenvertreters für eine Person, hinsichtlich derer die gesetzlichen Voraussetzungen nicht vorliegen, die also ihre Angelegenheiten selbständig, ggf mit Unterstützung besorgen kann, bildet einen Eingriff in das Persönlichkeitsrecht, das absoluten Schutz genießt. UE wären hier der betroffenen Person - wohl entgegen der hA ${ }^{17}$ - ideelle Schadenersatzansprüche zuzuerkennen, ${ }^{18}$ jedenfalls bei Vorsatz und grober Fahrlässigkeit. Dass die betroffene Person selbst jederzeit handeln kann, ändert am Umstand, dass Vertretungshandlungen für sie durch eine andere Person nach außen rechtswirksam gesetzt werden können, nichts. Relevant ist dieser Umstand zumindest bei der gerichtlichen Erwachsenenvertretung, die anders als die Vorsorgevollmacht, die gewillkürte bzw die gesetzliche Erwachsenenvertretung nicht durch einfachen Widerruf $(\mathbb{\$} 246$ Abs $1 \mathrm{Z} 3$ und 4) bzw durch einfachen Widerspruch der vertretenen Person zurückgenommen werden kann. Die Selbstbestimmung ( $\mathbb{S} 239)$ und der Nachrang der Stellvertretung (\$240) - beides Prinzipien des Persönlichkeitsrechts - wären bei zu weitreichender gerichtlicher Erwachsenenvertretung verletzt. Warum die Verletzung derart fundamentaler Prinzipien nur dann eine schadenersatzrechtliche Beachtung erfährt, wenn damit ein vermögensrechtlicher Schaden für den Betroffenen einhergeht, ist $\mathbf{u E}$ nicht nachvollziehbar. Lediglich wenn der Betroffene wegen seiner zu weitgehenden (und daher rechtswidrigen) Einschränkung körperliche Symptome mit Krankheitswert aufweist, sind ideelle Schadenersatzansprüche nach hA ${ }^{19}$ denkbar. Diese Situation ist uE unbefriedigend, da sich ein Kausalzusammenhang des genannten Umstands mit der Verschlechterung des Krankheitsbildes niemals nachweisen lassen wird.

16 Schaner in Deixler-Hübner/Schaner, HB Erwachsenenschutzrecht Rz 4.11, näher auch $4.80 \mathrm{ff}$.

17 Karner in $K B B^{5} \$ 1293 \mathrm{Rz} 2$; zu den Voraussetzungen der Zuerkennung von ideellen Schäden Wagner in Schwimann/Kodek, ABGB $\$ 1293 \mathrm{Rz} 23 \mathrm{ff}$.

18 So bereits im Grundsatz auch J. Ecker/Wagner in Sautner/Jesionek (Hrsg), Handbuch Opferrechte in europäischer, rechtsvergleichender und österreichischer Perspektive (2017) $320 \mathrm{ff}$.

$19 \mathrm{Vgl} \mathrm{RS0031111} \mathrm{zum} \mathrm{Ersatz} \mathrm{bei} \mathrm{Krankheitswert.}$ 
Die dargelegten Erwägungen lassen sich auf die Wirkungsbereiche in allen Kategorien der Erwachsenenvertretung übertragen. Diese dürfen nur noch beschränkt eingeräumt werden. Wegen der bestehenden Widerrufsmöglichkeit bzw Widerspruchsmöglichkeit des Schutzberechtigten - bei der Vorsorgevollmacht, der gewählten und gesetzlichen Erwachsenenvertretung - ist der Bereich der gerichtlichen Erwachsenenvertretung wohl am bedeutsamsten. Der Wirkungsbereich der Vorsorgevollmacht kann für einzelne Angelegenheiten oder für Arten von Angelegenheiten erteilt werden ( $\$ 261)$; für die gewählte Erwachsenenvertretung gilt gleiches gem $\$ 265$ Abs 3; bei der gesetzlichen Erwachsenenvertretung ist der Wirkungsbereich mit den taxativ genannten Vertretungshandlungen nach $\ 269$ Abs 1 umrissen. ${ }^{20}$ Die Befugnisse des gerichtlichen Erwachsenenvertreters werden nach neuer Rechtslage deutlich auf bestimmte Vertretungshandlungen beschränkt. Eine pauschale Übertragung aller Angelegenheiten ist nicht mehr möglich, vielmehr sieht $\ 272$ eine Bestellung nur für einzelne oder bestimmte Arten von Angelegenheiten, die gegenwärtig zu besorgen sind, vor. ${ }^{21}$

Auch wenn eine Stellvertretung nach außen unvermeidlich ist, soll nach der Bestimmung des $\mathbb{S} 241$ die Selbstbestimmung weitestmöglich erhalten bleiben: Ein Vorsorgebevollmächtigter oder Erwachsenenvertreter hat dafür zu sorgen, dass die vertretene Person im Rahmen ihrer Fähigkeiten und Möglichkeiten ihre Lebensverhältnisse nach ihren Wünschen und Vorstellungen gestalten kann und sie - soweit wie möglich - in die Lage zu versetzen, ihre Angelegenheiten selbst zu besorgen. Daher muss ein Vorsorgebevollmächtigter oder Erwachsenenvertreter die yertretene Person stets über seine beabsichtigten Entscheidungen rechtzeitig informieren und ihr die Möglichkeit geben, sich dazu in angemessener Frist zu äußern (Informations- und Wunschermittlungspflicht). ${ }^{22}$ Dies gilt nicht nur bei wichtigen, sondern gerade auch bei geringfügigen Angelegenheiten. Außer in Fällen, bei denen das Wohl des Vertretenen hierdurch erheblich gefährdet wäre, sind der Wille und die Meinung des Vertretenen vom Vertreter jedenfalls zu berücksichtigen. Aus diesen Grundsätzen ergibt sich auch eine Zweifelsregel zugunsten der Wünsche des Vertretenen. Diese Anordnung ist allerdings - ausweislich der $\mathrm{Mat}^{23}$ - eine reine Innenvorschrift, sodass Rechtsgeschäfte, die der Vertreter für die schutzberechtigte Person abschließt, trotz der Missachtung von Wünschen der Betroffenen wirksam sind. ${ }^{24} \mathrm{Ob}$ sich aus der Missachtung des Willens der betroffenen Person Schadenersatzpflichten ergeben können, ist fraglich. Setzt man nämlich voraus, dass die betroffene Person über die nötige

20 Dazu im Detail: Schaner in Deixler-Hübner/Schaner, HB Erwachsenenschutzrecht $\mathrm{Rz} 4.10 \mathrm{ff}$.

$21 \mathrm{Vgl}$ Schweighofer, Einwilligung in die medizinische Behandlung, EF-Z 2018/84; OGH 13.3.2018, $5 \mathrm{Ob} 12 / 18 \mathrm{~b}$.

22 Zierl/Schweighofer/Wimberger, Erwachsenenschutzrecht ${ }^{2}$ Rz 144.

23 ErläutRV 1461 BlgNR 25. GP, 20.

24 Weitzenböck in Deixler-Hübner/Schawer, HB Erwachsenenschutzrecht Rz 6.25. 
Geschäftsfähigkeit selbst nicht verfügt, so könnte man unterstellen, dass sie auch nicht die Einsichtsfähigkeit in komplexe Angelegenheiten der Vermögenssorge hat. $\ 241$ Abs 3 spricht aber genau davon, nämlich dass die Äußerung der vertretenen Person zu berücksichtigen ist, es sei denn, ihr Wohl wäre hierdurch erheblich gefährdet.

Beispiel: Der demente Bauer B wünscht sich eine Verpachtung seines Gutes an einen Gnadenhof zu niedrigem Zins. Der Erwachsenenvertreter kennt den Wunsch des Bauern B, verpachtet aber das Grundstück an den Agrarbauern A, der einen höheren Pachtzins zu zahlen bereit ist.

Das Bsp zeigt das Dilemma zwischen der Wunschermittlungspflicht, die grundsätzlich auch unter wirtschaftlichen Aspekten „unvernünftige“ Geschäfte deckt, den Rechnungslegungspflichten des Erwachsenenvertreters und den gerichtlichen Überwachungspflichten. Nimmt man die Aussage in den $\mathrm{Mat}^{25}$ ernst, wonach es zur persönlichen Autonomie gehört, auch „Entscheidungen nach seinem eigenen, von allgemeinen Vorstellungen abweichenden Wertesystem zu treffen", so erscheint es nur konsequent und schon dem Wortlaut des $\ 241$ Abs 3 geschuldet, wenn die persönliche Autonomie in solchen Fragen von allen Seiten (Vertreter, Gericht) tatsächlich gelebt wird: Nur wenn eine erhebliche Gefährdung des Wohls der betroffenen Person besteht und ihr „Wille“ durch die psychische Krankheit verzerrt ist, darf davon abgewichen werden. Auch der OGH hatte bereits vor Inkrafttreten des 2. ErwSchG iZm der pflegschaftsgerichtlichen Genehmigung von Rechtsgeschäften klargestellt, dass nicht allein materielle Gesichtspunkte maßgebend sind, sondern dass auch die Interessen und Wünsche des Pflegebefohlenen, aber auch seien Befindlichkeit und seine konkreten Lebensumstände berücksichtigt werden müssen. Auch die mögliche Verschlechterung der emotionalen Befindlichkeit ist in eine von den Umständen des Einzelfalls abhängige Gesamtbeurteilung einzubeziehen. ${ }^{26}$

Im genannten Bsp werden dem Schutzbefohlenen wohl nach hA keine Schadenersatzansprüche wegen Missachtung seines Willens zuzuerkennen sein. ${ }^{27}$ Materiell hat B einen Zuwachs erfahren, ein Vermögensschaden liegt daher nicht vor. Anders ist die Situation jedoch zu beurteilen, wenn tatsächlich aufgrund der Missachtung des Willens des Vertretenen ein Vermögensschaden eintritt. Schon nach den Regeln des Auftragsverhältnisses ergibt sich, dass bei Verschulden des Gewalthabers der Vermögensschaden ersatzfähig ist. ${ }^{28}$

25 ErläutRV 1461 BlgNR 25. GP, 19 (zu \240).

264 Ob 146/16v; 5 Ob 210/17v.

27 Zum Problem von Interessenskonflikten auch Weitzenböck in Deixler-Hübner/Schauer, HB Erwachsenenschutzrecht Rz $6.47 \mathrm{ff}$.

$28 \mathrm{Vgl}$ auch P. Bydlinski, KBB S $1012 \mathrm{Rz}$ 1; Bollenberger in Deixler-Hübner/Schaner, HB Erwachsenenschutzrecht Rz 10.13 und 10.21. 
Beispiel: Der aufgrund einer geistigen Behinderung schutzberechtigte X ersucht anlässlich einer Charity-Verkaufsveranstaltung zugunsten behinderter Personen seinen Erwachsenenvertreter (E), ihm ein von seinem Freund, dem ebenso behinderten fußmalenden Künstler K, gemaltes Bild um $€ 100$,- zu kaufen. Der Erwachsenenvertreter verweigert dies, da er den Ankauf als nutzlos erachtet. Die Anschaffung wäre finanziell für X durchaus tragbar. Auch als X selbst das Bild mit dem ihm zur Verfügung stehenden Geld erwerben will, unterbindet E den Erwerbsvorgang. Kurze Zeit später wird K berühmt, seine Gemälde sind nur mehr im „4-stelligen Bereich“ erhältlich.

Im Beispiel hat der Erwachsenenvertreter die Wunschberücksichtigungspflicht verletzt, woraus ein Vermögensschaden resultiert. Sofern daraus resultierende Vermögensschäden nicht außerhalb der allgemeinen Lebenserfahrung liegen - das sei im Bsp dahingestellt - wird man schon nach $\$ 249$ eine Schadenersatzpflicht bejahen können. Der OGH hat dazu bereits vor Inkrafttreten des 2. ErwSchG ausgeführt, dass die Frage, ob einem Sachwalter, $\mathrm{zu}$ dessen Aufgabenbereich auch die Vermögensverwaltung der betroffenen Person gehört, eine schuldhafte Verletzung seiner Pflichten, die zu einer Schädigung eines Vermögenswerts der betroffenen Person führt, vorzuwerfen ist, nur nach den Umständen des konkreten Einzelfalls zu beurteilen ist. ${ }^{29}$

Generell unklar - sowohl aus Sicht des Persönlichkeitsrechts als auch des Haftungsrechts - ist die Situation, gerade im Lichte des Art 12 der UN-Behindertenkonvention, wenn die betroffene Person die ihr angebotene Unterstützung nicht annimmt oder nicht mehr annehmen kann. Die ErläutRV ${ }^{30}$ gehen davon aus, dass in solchen Fällen und innerhalb enger Grenzen weiterhin die Möglichkeit bestehe, dass gerichtlich bestellte Vertreter Entscheidungen treffen, um die betroffene Person yor einer Selbstschädigung zu schützen. Jedenfalls dann, wenn eine gerichtlich begründete Erwachsenenvertretung zum Wohl des Betroffenen ausgeübt wird, scheiden Schadenersatzansprüche aus. Die Autonomie des Betroffenen tritt seinetwegen selbst in den Hintergrund. Umso wichtiger ist in diesen Fällen die Kontrolltätigkeit des Gerichts. ${ }^{31}$

Das neue Erwachsenenschutzrecht führt zu einer veränderten und verstärkten Kontrolle durch das Gericht (\$259). Dies sowohl hinsichtlich der persönlichen Pflichten des Erwachsenenvertreters als auch hinsichtlich der Vermögenssorge. Die Kontrolltätigkeit des Gerichts ist hoheitlich und kann daher haftungsrechtlich unter dem Blick des Amtshaftungsrechts gesehen werden (dazu näher unten). Legt der Erwachsenenvertreter Rechnung, so muss das Gericht prüfen und nötigenfalls Aufträge erteilen $(\mathbb{S} 259) .{ }^{32}$

$295 \mathrm{Ob} \mathrm{210/17v,} \mathrm{RS0049179.}$

30 ErläutRV 1461 BlgNR 25. GP, 19.

31 Schaner in Deixler-Hübner/Schaner, HB Erwachsenenschutzrecht Rz 4.72 ff;

32 Eingehend dazu Weitzenböck in Deixler-Hübner/Schaner, HB Erwachsenenschutzrecht Rz 1.106 ff; Zierl/Schweighofer/Wimberger, Erwachsenenschutzrecht ${ }^{2}$ Rz 305 ff. 


\section{B. Die Unterscheidung zwischen Außen- und Innenverhältnis und deren haftungsrechtliche Relevanz}

Anknüpfend an die eingangs dargelegten Bemerkungen und Beispiele lässt sich sohin konstatieren, dass mit Bestellung zum Vorsorgebevollmächtigten und Erwachsenenvertreter aber va auch Pflichten einher gehen, deren Verletzung zu Haftungsfolgen des Vorsorgebevollmächtigten oder Erwachsenenvertreters führen können.

Hinsichtlich der Haftungsfolgen ist zwischen Außen- und Innenverhältnis zu unterscheiden: Das Innenverhältnis betrifft die Haftung des Erwachsenenvertreters gegenüber der schutzberechtigten Person, im Außenverhältnis kann sich eine Haftung des Erwachsenenvertreters im Zusammenhang mit seiner Vertretungstätigkeit Dritten gegenüber ergeben. Ansonsten bleibt es im Außenverhältnis bei den schon oben getroffenen Feststellungen: Ob sich aus den Regeln des Erwachsenenschutzrechts gegenüber Dritten Schutzpflichten ableiten lassen, lässt sich nur mit Blick auf den Rechtswidrigkeitszusammenhang klären.

\section{Innenverhältnis}

Erwachsenenvertreter und Vorsorgebevollmächtigte haften der vertretenen Person gegenüber gem $\mathbb{2} 249$ Abs 1 (alte Rechtslage: $\$ 277)^{33}$ für ihr Handeln. Nach den Mat zur alten RL soll damit die Anwendbarkeit allgemeinerer schadenersatzrechtlicher Grundsätze zum Ausdruck gebracht werden. ${ }^{34}$ Diese Aussage ist zwar nicht falsch, lässt sich uE aber präzisieren: Verstöße gegen die Rechte der schutzbedürftigen Person sind Verstöße nicht bloß gegen deliktische Pflichten, sondern solche aus einem gesetzlichen Sonderrechtsverhältnis. Dieses fwird durch die gesetzlichen Normen über die Vorsorgevollmacht und Erwachsenenyertretung konkretisiert. Ein solches Verhältnis wird den Regeln ex contractu zu unterstellen sein. ${ }^{35}$ In diesem Rahmen sind auch Vermögensschäden ersatzfähig. Im Rahmen der gewählten Erwachsenenvertretung liegt zudem eine ausdrückliche Vereinbarung $(\$ 265)$ vor, ${ }^{36}$ sodass das Innenverhältnis jedenfalls als ex contractu anzusehen ist. Sowohl im Rahmen des gesetzlichen Sonderrechtsverhältnisses als auch auf Rechtsver-

33 Zur unveränderten Übernahme der in L und Rsp entwickelten Grundsätze für alle Vertreter auf die neue RL siehe Weitzenböck in Deixler-Hübner/Schaner, Erwachsenenschutzrecht. Beiträge zum 2. Erwachsenenschutzgesetz 39.

34 ErläutRV 296 BlgNR 21. GP, 77.

35 Wagner in Schwimann/Kodek, ABGB $\$ 1298 \mathrm{Rz} 9 \mathrm{ff}$; Kodek in Kletečka/Schauer, ABGB-ON ${ }^{1.03} \$ 1298 \mathrm{Rz} 4 \mathrm{ff}$.

36 Schaner in Deixler-Hübner/Schaner, HB Erwachsenenschutzrecht Rz 4.32 ff. 
hältnisse ex contractu findet $\ 1298$ Anwendung; dazu näher noch unter III. B.4. ${ }^{37}$

Die in der Rsp entschiedenen Fälle betrafen va Vermögensschäden, derentwegen die Haftung des Sachwalters schlagend wurde:

In $4 \mathrm{Ob} 26 / 10 t$ hatte es der Sachwalter unterlassen, einen Anspruch auf Waisenpension geltend zu machen. Der OGH legte zu Recht dar, dass es zu den Pflichten eines Sachwalters gehöre, den Vermögensstand des Betroffenen gründlich zu erforschen und regelmäßig zu prüfen, ob aufgrund veränderter Umstände neue Einkommens- und Leistungsansprüche des Betroffenen entstanden sind.

In der $\mathrm{E} 1 \mathrm{Ob}$ 97/12i wurde die einsteilige Sachwalterin mit der Vermögensverwaltung der Betroffenen betraut, wozu auch ein Wertpapierdepot gehörte. Als haftungsbegründender Umstand wurde ua die Umwandlung von nicht mündelsicher veranlagten Wertpapieren in mündelsichere ( $\$ 230 \mathrm{~b})$, vorgebracht; diesem Vorwurf blieb aber der Erfolg versagt.

In der E 10 Ob 17/17y wurde die Sorgfaltswidrigkeit des Sachwalters, der verjährte Forderungen nicht einklagte, verneint. Ein zum Sachwalter bestellter Rechtsanwalt muss die Interessen des Betroffenen bestmöglich wahren. Dazu zählt die Verpflichtung, vorhandenes Vermögen soweit wie möglich zu vermehren oder zumindest zu erhalten, jedenfalls aber nicht unnötig zu verringern. Das mit einem entsprechenden Prozesskostenrisiko verbundene Einklagen verjährter Ansprüche widerspreche diesem Ziel eindeutig.

Wesentlich in diesem Zusammenhang ist, dass sich die Sorgfaltspflichten des Erwachsenenvertreters am Wohl der vertretenen Person orientieren und dabei - wie bereits oben unter dem Aspekt der Selbstbestimmung dargelegt (siehe I.A), siehe dazu auch noch näher unten - die subjektiven Interessen und Wünsche der schutzberechtigten Person gem $\mathbb{} \$ 239 \mathrm{Abs} 1$ zu berücksichtigen sind. Das gilt auch für die Vermögenssorge. Hier ist mit $\ 258 \mathrm{nF}$ insofern eine Änderung eingetreten, als die angemessene Befriedigung der Bedürfnisse der schutzberechtigten Person jedenfalls im Zentrum steht. Das Ansparziel von Vermögenswerten ist nachgeordnet.

Bsp: Besteht eine durchschnittliche Rentnerin, die nunmehr dement ist, darauf, täglich nachmittags ins Kaffeehaus zu gehen, so ist ihr dieser Wunsch zu „gönnen“; anderes wäre wohl bei regelmäßigen Casinobesuchen der Fall. Auch Geldgeschenke an nahe Angehörige können gerechtfertigt sein. ${ }^{38}$

Hinsichtlich der Personensorge normiert $\mathbb{2 5 1}$, dass ein Erwachsenenvertreter nicht zur Betreuung der vertretenen Person verpflichtet ist. Ist sie aber nicht umfassend betreut, so hat er sich, unabhängig von seinem Wirkungsbereich darum zu bemühen, dass ihr die gebotene medizinische und soziale Betreuung gewährt wird. Betreuung meint all jene Handlungen, die

37 Wagner in Schwimann/Kodek, $\mathrm{ABGB}^{4} \$ 1298 \mathrm{Rz} 2 \mathrm{ff}$; vgl auch Bollenberger in DeixlerHübner/Schauer, HB Erwachsenenschutzrecht Rz 10.17.

38 Bollenberger in Deixler-Hübner/Schaner, HB Erwachsenenschutzrecht Rz 10.12. 
vornehmlich den persönlichen Lebensbereich betreffen und ohne die ein pflegebedürftiger Mensch der Verwahrlosung ausgesetzt wäre (bspw An- und Auskleiden, Körperpflege, Einnahme von Medikamenten). Eine solche Pflicht des Erwachsenenvertreters zur Bemühung um Gewährung medizinischer und sozialer Versorgung, soll es nur dann geben, wenn die vertretene Person „nicht umfassend betreut" ist. ${ }^{39}$ Die Mat geben dahingehend Auskunft, dass unter einer umfassenden Betreuung auf $\$ 27 \mathrm{~b}$ KSchG abzustellen sei, sodass sowohl für die Unterkunft der vertretenen Person als auch für Betreuung und Pflege gesorgt ist. Nicht darunter fallen die Tagesstätte oder die Betreuung durch eine Heimhilfe. ${ }^{40}$ Selbst wenn der Erwachsenenvertreter nur für eine einzige Angelegenheit eingesetzt ist, trifft ihn eine Bemühungspflicht. Dies gilt nicht für den Vorsorgebevollmächtigten, die persönliche Betreuung kann aber vereinbart werden. Fraglich ist, wann eine Verletzung der Bemühungspflicht angenommen werden kann. Die Formulierung deutet jedenfalls auf eine bloße Sorgfaltsverbindlichkeit hin, wobei wohl in allen Kategorien, die einer Vereinbarung zugänglich sind (also Vorsorgevollmacht und gewählte Erwachsenenvertretung), die Betreuung auch als Erfolgsverbindlichkeit sichergestellt werden kann. Jedenfalls wenn kein Bemühen bzgl der Personensorge festgestellt werden kann und der Person daraus ein Schaden an der körperlichen Integrität erwächst, sind Haftungsfolgen naheliegend. Anders ist die Situation aber dann, wenn etwa der Wunsch der betroffenen Person gerade in der Bewahrung weitestmöglicher Autonomie liegt: Fehleinschätzungen ex ante, die letztlich aber einen Schaden an der schutzberechtigten Person oder an ihren Vermögenswerten herbeiführen (Bsp: Person mit Demenz im Anfangsstadium vergisst, den Herd abzuschalten), münden nicht „automatisch“ in Haftungsfolgen. Insofern ist die neue Autonomie haftungsrechtlich konsequent umzusetzen. Sie kann nicht nur als haftungsbegründend, sondern auch als haftungsentlastend gesehen werden.

\section{Außenverhältnis: Haftung des Erwachsenenvertreters Dritten gegenüber}

Erwachsenenvertreter und Vorsorgebevollmächtigte nehmen in Wahrnehmung der Personensorge auch Vertretungsaufgaben wahr: Sie agieren als Stellvertreter der schutzberechtigten Person. Eine Haftung des Erwachsenenvertreters Dritten gegenüber ergäbe sich bereits aus Regeln des allgemeinen

39 RV 1461 25. GP, 29 (zu \251); auch Deixler-Hübner in Deixler-Hübner/Schaner, HB Erwachsenenschutzrecht Rz 7.8 f; Zierl/Schweighofer/Wimberger, Erwachsenenschutzrecht ${ }^{2} \mathrm{Rz} 242$.

40 RV 1461 25. GP, 29 (zu $\ 251$ ); auch Deixler-Hübner in Deixler-Hübner/Schaner, HB Erwachsenenschutzrecht Rz 7.11.; Zierl/Schweighofer/Wimberger, Erwachsenenschutzrecht ${ }^{2} \mathrm{Rz} 243$. 
Vertretungsrechts (siehe dazu a). Auf die Besonderheiten der Erwachsenenvertretung gilt es dabei allerdings einen Blick zu werfen (siehe dazu b).

\section{a) Grundsätze des Vertretungsrechts}

Das ABGB regelt das Vertretungsrecht im zweiundzwanzigsten Hauptstück unter dem Titel „Von der Bevollmächtigung und anderen Arten der Geschäftsfübrung“. Die dortigen Regelungen trennen nicht scharf zwischen Innen- und Außenverhältnis, wenngleich die heutige Dogmatik eine solche Trennung vorgibt. ${ }^{41}$ Obwohl die Regelungen des ABGB va das rechtsgeschäftliche Vertretungsrecht darlegen, gelten diese Grundsätze auch in Fällen, in denen das Gesetz, Gerichtsentscheid oder Organbestellung eine Vertretung vorsehen. ${ }^{42}$ Auch im vorliegenden Zusammenhang wird man auf die Grundsätze abzustellen haben, wobei allerdings die Besonderheiten der Materie, die mit dem 2. ErwSchG eingeführt wurden, durchschlagen.

Aus der Missachtung von Regelungen bzgl Auftrag, Bevollmächtigungsvertrag, Dienstvertrag ergibt sich zum einen eine Haftung im Innenverhältnis: Der Geschäftsbesorger haftet nach $\ 1012$ für jeden Schaden, der durch eine von ihm verschuldeten Verletzung der ihm im Zuge der Geschäftsbesorgung obliegenden Pflichten entstanden ist. Die Übertragung dieses Prinzips auf die Zusammenhänge des Erwachsenenschutzes erfolgte bereits oben (B.1).

Bei Überschreitung der Vollmacht normiert \$1009, dass der Gewalthaber für die Folgen haftet. Der Vollmachtsumfang eines Bevollmächtigungsvertrages bestimmt sich nach allgemeinem Zivilrecht durch die Auslegung der Willenserklärung des Geschäftsherrn, aber auch aus dem Gegenstand und der Natur des Geschäftes. ${ }^{43}$ Im Außenverhältnis wird der Stellvertreter dem Dritten gegenüber bei gescheiterter Vertretung gem $\ 1019$ haftbar werden, wobei das Vértrauensinteresse diesfalls mit dem Erfüllungsinteresse begrenzt ist.

\section{b) Begrenzung der cic durch den Schutzzweck des ErwachsenenschutzG}

Inwiefern \1019 bei Fällen der Überschreitung der Vertretungsmacht ebenso bei der Erwachsenenvertretung zur Haftung führt, ${ }^{44}$ bedarf $\mathrm{uE}$ ganz genauer Betrachtung. Vielmehr scheint es so zu sein, dass bedingt durch die Grundwertung der Selbstbestimmung und Autonomie oftmals in Fällen, die nach

41 Apathy in Schwimann/Kodek, ABGB $4 \mathbb{4} 1002 \mathrm{Rz} 3 ;$ P. Bydlinski in KBB $51002 \mathrm{Rz} 3$; Riedler, Zivilrecht III, Schuldrecht Besonderer Teil, Vertragliche Schuldverhältnisse ${ }^{6}$ $\mathrm{Rz}$ 9/1.

42 Apathy in Schwimann/Kodek, ABGB $\$ 1002 \mathrm{Rz} 2$; P. Bydlinski in $K B B^{5} \$ 1002 \mathrm{Rz} 9 \mathrm{ff}$.

43 P. Bydlinski in $K B B^{5} \$ 1009$ Rz 1 f.; Riedler, Zivilrecht III, Schuldrecht Besonderer Teil, Vertragliche Schuldverhältnisse ${ }^{6}$ Rz 9/9.

44 Dies bejahend Bollenberger in Deixler-Hübner/Schauer Rz 10.10. 
allgemeinen Grundsätzen als „falsus procurator“ zur Haftung führen könnten, mangels Rechtswidrigkeit des Vertreters keine Haftung besteht. Der Vertrauensschutz für Dritte ist im Bereich des Erwachsenenschutzes relativiert.

\section{Zur haftungsrechtlichen Relevanz der Pflichten des Erwachsenenvertreters}

\section{A. Vertretungshandlungen iZm Personensorge}

IZm der Personensorge gilt, dass gem $\ 250$ Abs 1 Z 4 ein Vorsorgeberechtigter nur dann Vertretungshandlungen setzen darf, wenn diese von seinem Wirkungsbereich umfasst sind, die vertretene Person nicht entscheidungsfähig ist und das Wohl einer vertretenen Person die Vertretung erfordert. Auch in diesem Zusammenhang ist vorerst abzuklären, ob die Vertretungshandlung in den Rahmen des Wirkungsbereichs des Vertreters nach dem 4-Säulen-Modell fällt $(\mathbb{S}$ 261, 265, 269, 272).

Gibt die vertretene Person zu erkennen, dass sie die geplante Vertretungshandlung ablehnt, so hat diese bei sonstiger Rechtsunwirksamkeit zu unterbleiben, es sei denn, das Wohl der vertretenen Person wäre sonst erheblich gefährdet. Ausweislich der Mat bewirkt der Widerspruch, dass auch eine erforderliche Vertretungshandlung zu unterbleiben hat; ${ }^{45}$ nur wenn das Wohl der vertretenen Person eine erhebliche Gefährdung nahelegt, darf trotz Widerspruchs gehandelt werden. Schon in diesem Zusammenhang zeigt sich, dass nach dem Schutzzweck der Regeln über die Erwachsenenvertretung das Außenverhältnis in besonderem Maße vom Innenverhältnis abhängt. Die Nichtigkeitsanordnung von Vertretungshandlungen, die die schutzberechtigte Person nicht wünscht, sichert die Autonomie. Der Ableitung von Ansprüchen Dritter, wie etwa $\$ 1019$ solche vorsieht, ist somit mit äußerster Vorsicht zu begegnen. Denkbar wäre, dass in solchen Fällen den Erwachsenenvertreter Aufklärungspflichten bzgl der allenfalls fehlenden Bindungswirkung seiner Erklärung treffen. Nur wenn man solche klar bejahen kann, lassen sich die Haftungsfolgen der cic konstatieren.

Nach $\$ 250$ Abs 3, der dem bisher geltenden $\$ 275$ Abs 2 S 1 entspricht, hat ein Erwachsenenvertreter (nicht aber Vorsorgebevollmächtigter) in wichtigen Angelegenheiten die Genehmigung des Gerichts einzuholen. Dies gilt nicht bei Gefahr in Verzug, wenn also das Wohl erheblich gefährdet wäre. Diesfalls ist die Vertretungshandlung bis zur gerichtlichen Genehmigung schwebend unwirksam. Wichtige Angelegenheiten der Personensorge können insb einige der in $\ 167$ Abs 2 genannten Angelegenheiten sein. ${ }^{46}$ Auch daraus kann sich

45 RV 1461 25. GP, 28 (zu \250).

46 ErläutRV1461 25. GP, 29. 
eine Aufklärungspflicht gegenüber Dritten ergeben. Der Umstand der Vertretung trotz nachträglicher Nichtgenehmigung des Gerichts bildet keinesfalls ein Indiz für eine objektive Sorgfaltswidrigkeit des Erwachsenenvertreters. Die Frage der Genehmigungsfähigkeit richtet sich allein nach dem Wohl der schutzberechtigten Person, entfaltet keine Außenwirkung und unterliegt allein den gerichtlichen Maßstäben.

\section{B. Vertretungsangelegenheiten in Vermögensangelegenheiten}

$\mathrm{Zu}$ unterscheiden ist zwischen Vertretungsangelegenheiten, die zum ordentlichen Wirkungsbereich gehören und jenen, die nicht mehr zum ordentlichen Wirkungsbereich gehören.

\section{Grundsätzliches zur Vertretung im Rahmen des 4-Säulen- Vertretungsmodells}

Wie bereits dargelegt, nehmen schutzberechtigte Personen am Rechtsverkehr durch den Vorsorgebevollmächtigten bzw Erwachsenenvertreter teil. Die maßgebliche Änderung des 2. ErwSchG ist nun, dass nach dem Prinzip der Autonomie auch im Rahmen einer Vertreterbestellung idR (außer bei Genehmigungsvorbehalten, siehe dazu unten (B.2.g)) die betroffene Person ihre Geschäftsfähigkeit grundsätzlich nicht verliert: Dieses Postulat ergibt sich aus Art 12 UN-Behindertenkonvention, wonach Ö verpflichtet ist, alle notwendigen Maßnahmen zu ergreifen, behinderten Menschen Unterstützung zur Ausübung der Rechts- und Handlungsfähigkeit zukommen zu lassen. Eine pauschale Vertretung für alle Angelegenheiten soll es nicht mehr geben. Vertretungsrechtlich ergeben sich mit dem 2. ErwSchG folgende „Besonderheiten“:

Im Erwachsenenschutzrecht sind die vom Vertreter auszuübenden Aufgaben konkret je nach Vertretungsart und Wirkungsbereich zu bestimmen. Die fehlerhafte Ausübung einer bestehenden Vertretungsmacht führt im Innenverhältnis - wie bei der Schadenshaftung des Geschäftsbesorgers gem $\$ 1009$ - zur Verschuldenshaftung $(\$ 249)^{47}$ und nach uE richtiger Ansicht zur Anwendung der Regelungen der Beweislastumkehr gem $\mathbb{\$} 1298 .{ }^{48}$ Auf den Umstand, dass auch Handlungen innerhalb des Wirkungsbereichs, die von einer Vollmacht gedeckt sind, die aber unter Missachtung der Wunschermittlungspflicht gesetzt wurde, Schadenersatzpflichten gegenüber dem/der Betroffenen auslösen können, wurden bereits oben hingewiesen.

47 Kathrein in Deixler-Hübner/Schaner, HB Erwachsenenschutzrecht Rz 1.44.

48 IdS bereits Strasser in Rummel, $\mathrm{ABGB}^{3} \$ 1012 \mathrm{ABGB} \mathrm{Rz} 7$. 